\title{
Revisión de las Propiedades de Psicométricas de la Escala de Evaluación de Riesgos Psicosociales en el Trabajo SUCESO/ ISTAS 21 en el Contexto Chileno
}

\author{
A REVIEW OF THE PSYCHOMETRIC PROPERTIES OF THE PSYCHOSOCIAL RISK FACTORS IN THE WORKPLACE \\ SCALE SUSESO/ISTAS 21 IN THE CHILEAN CONTEXT
}

Guido Cerda-Silva1', Fidel Porras-Tapia ${ }^{1}$

1. Universidad Arturo Prat Iquique Chile.

\begin{abstract}
RESUMEN
La implementación del Cuestionario SUSESO/ISTAS 21 como un protocolo de vigilancia de salud mental en las organizaciones ha sido utilizada en los últimos cinco años en Chile. El objetivo de esta investigación es analizar el comportamiento de las propiedades psicométricas del cuestionario en su versión breve en el contexto chileno. El estudio realizó un análisis factorial confirmatorio con cinco dimensiones. Se emplearon medidas de bondad para ajuste del modelo como RMR, GFI, AGFI, NFI y PGFI. Para el la obtención de la fiabilidad del instrumento se emplea el Coeficiente Omega. De igual manera, se realizó un análisis de la validez convergente y discriminante del cuestionario. Por su parte, la muestra está compuesta por 1184 trabajadores. Se concluye que el cuestionario SUSESO/ISTAS 21 en su versión breve no cuenta con los parámetros de confiabilidad y validez necesarios para su aplicación. Finalmente, la estrategia de reducción de dimensiones no es apropiada.
\end{abstract}

(Cerda-Silva G, Porras-Tapia F, 2018. Revisión de las Propiedades de Psicométricas de la Escala de Evaluación de Riesgos Psicosociales en el Trabajo SUCESO/ISTAS21 en el Contexto Chileno. Cienc Trab. SepDic; 20 [63]: 121-125).

Palabras clave: FACTOR DE RIESGO, VALIDEZ, CONFIABILIDAD.

\section{ABSTRACT}

In the last five years, SUSESO/ISTAS21 has been implemented as the main protocol for watching mental health within organizations in Chile. The present work aims to review the psychometric properties of the short form of this questionnaire in the Chilean context. For this purpose we conducted a confirmatory factorial analysis (CFA) for a five-factor model, checking for goodness of fit (through GFI, AGFI, PGFI, NFI, and RMR), on a sample of 1.184 workers. Additionally, the reliability of the instrument was assessed through the Omega Coefficient, and the convergent and discriminant validity were also checked. These multiple analyses show that the short form SUSESO/ ISTAS21 questionnaire does not meet the realiability and validity standards required for its use. The former reveals the inadequacy of the dimension reduction strategy implemented.

Key words: RISK FACTOR, VALIDITY, RELIABILITY, SUSESO/ISTAS21.

\section{INTRODUCCION}

Los espacios laborales a nivel mundial han iniciado un proceso de cambio ${ }^{1}$, así como la forma en cómo se concibe al trabajador en el contexto profesional. Desde los años de la revolución industrial a la actualidad, las condiciones de trabajo ${ }^{2}$ han sido una preocupación para la Organización Internacional del Trabajo $(\mathrm{OIT})^{3}$, la Organización Mundial de la Salud (OMS) y la

Correspondencia / Correspondence:

Ps. Mg. Guido Cerda Silva

Facultad de Ciencias de la Salud Universidad Arturo Prat

Av. Arturo Prat 212. Iquique.

Tel.: (57-2) 526711

e-mail: gucerda@unap.cl

Recibido: 10 de agosto de 2018 / Aceptado: 07 de noviembre de 2018
Organización de Naciones Unidas (ONU), al igual que los horarios de empleo, tendencias salariales ${ }^{4}$, enfermedades de salud física ${ }^{5} \mathrm{y}$ mental ${ }^{6}$ generadas por el contexto laboral.

En Chile, se han realizado distintos estudios para fortalecer la incidencia de las condiciones de trabajo favorables para la salud del trabajador $^{7}$, pero no es hasta el año 2013 que la Ley $16.744^{8}$ de enfermedades profesionales y accidentes laborales otorga un respaldo legal explícito ${ }^{9}$, incorporando regulaciones dirigidas a velar por la salud mental de los trabajadores dentro las organizaciones ${ }^{1}$. a través de la Superintendencia de Seguridad Social (SUSESO).

Por medio de la Ley $\mathrm{N}^{\circ} 16.395 \mathrm{y}$ el artículo $12^{\circ}$ de la Ley $\mathrm{N}^{\circ} 16.744$, el DS 10. de 1968 y lo normado en la Resolución Exenta $N^{\circ} 336$, de 2013, del Ministerio de Salud, que aprobó el Protocolo de Vigilancia de Riesgos Psicosociales en el Trabajo, se establece la medición de los riesgos psicosociales (RPS) utilizando la validación Chilena Cuestionario SUSESO/ISTAS 21. ${ }^{11,12}$

Este instrumento ha sido construido con un riguroso diseño metodológico ${ }^{13}$, adaptado en distintos países, hasta llegar a Latinoamérica. El Copenhagen Psychosocial Questionnaire (CoPsoQ) $)^{14,15}$, desarrollado por el Instituto Nacional de Salud Ocupacional de Dinamarca, gracias a los trabajos de Kristensen, quien en el año 2000 identifica 
factores como condiciones del riesgo laboral para la salud. Posteriormente, en España, el Instituto Sindical de Trabajo, Ambiente y Salud (ISTAS) adapta la versión corta del CoPsoQ ${ }^{16}$, impulsando actividades para la mejora de las condiciones del trabajo y la promoción de la salud de los trabajadores.

Posteriormente comienza el proceso en Latinoamérica, las distintas institucionalidades generan normas para la gestión del riesgo psicosocial; así es como Chile, Argentina ${ }^{17,18}$ Colombia $^{19}$ y Perú ${ }^{2.21}$ desarrollan de forma voluntaria la medición de los factores de riesgo psicosociales. La Tabla 1 permite revisar el avance que ha tenido dicho proceso de medición RPS en Latinoamérica.

Tabla 1.

Evaluación temporal de la elaboración de escalas para medir riesgos psicosociales.

$\begin{array}{lll}\text { País } & \text { Año } & \text { Escala } \\ \text { Dinamarca } & 2000 & \text { Copenhagen Psychosocial Questionnaire } \\ \text { España } & 2003 & \text { CoPsoQ-ISTAS21 } \\ \text { Colombia } & 2008 & \text { Batería de Instrumentos para la medición de FRPS } \\ \text { Chile } & 2013 & \text { SUSESO/ISTAS21 } \\ \text { Perú } & 2014 & \text { Informe Técnico de RPS } \\ \text { Argentina } & 2015 & \text { CoPsoQ-ISTAS21 }\end{array}$

Con la implementación del Protocolo de Vigilancia de Riegos Psicosociales en el Trabajo, por medio de la medición de factores de riesgos, se busca identificar en las labores cotidianas del trabajador cuáles son los niveles de carga mental, así como las implicancias que esta tiene en su vida personal ${ }^{22}$ y el riesgo para su salud. ${ }^{23}$

Al ser utilizado el Protocolo en Chile desde el año 2014 en su legislación, esta investigación tiene por objetivo analizar el comportamiento de las propiedades psicométricas del Cuestionario SUSESO/ISTAS 21 en el contexto chileno, entregando antecedentes de los resultados que obtienen las empresas y organizaciones para la toma de decisiones en el ámbito de la salud mental.

La medición se realiza por medio de un cuestionario, el cual cuenta con dos versiones: una extensa de 142 preguntas y una versión breve con un total de 20 preguntas. En Chile, para su versión abreviada, se ha definido un total de 5 dimensiones que dan cuenta de un factor de riego particular, denominadas: Exigencias Psicológicas; Trabajo activo y posibilidades de desarrollo; Apoyo social en la empresa y calidad de liderazgo; Compensaciones y, finalmente, Doble Presencia.

\section{Material y Métodos}

El proceso de recopilación de datos se realiza con una institución pública de educación superior que se encuentra en etapa de aplicación del cuestionario SUSESO/ISTAS 21 en su versión breve. La institución brinda los datos de sus resultados para el análisis de las propiedades psicométricas del cuestionario mediante una solicitud formal resguardando la privacidad de los participantes y los nombres de las organizaciones.

\section{Participantes}

La muestra final fue de 1184 trabajadores. Los colaboradores participaron de manera voluntaria y anónima en el proceso de aplicación, cumpliendo con la rigurosidad del protocolo, según la legislación vigente en Chile.

\section{Descripción del Instrumento}

El cuestionario SUSESO/ISTAS 21 versión breve fue construido a partir de las preguntas que explicaban la mayor proporción de la varianza de las cinco dimensiones evaluadas por la versión extensa del instrumento. ${ }^{11}$ Se utiliza como filtro inicial o tamizaje, permitiendo establecer una visión general de los niveles de riesgos psicosociales laborales y facilitar su seguimiento en el tiempo.

La versión breve consta de dos secciones una general destinada a la caracterización sociodemográfica y una específica cuyos ítems dan cuenta de los cinco factores de riesgo, distribuidos como se muestra en la Tabla 2. El formato de respuesta empleado es una escala Likert de 5 puntos en función de la frecuencia (en sentido favorable desde $0=$ nunca, a $4=$ siempre y en sentido inverso desde $0=$ siempre, a 4 = nunca).

Tabla 2.

Dimensiones y Sub-dimensiones del Cuestionario SUSESO/ISTAS 21 versión completa.

$\begin{array}{llc}\text { Factor de riesgo psicosocial } & \text { Subdimensiones } & \text { Ítems } \\ & \text { Exigencias cuantitativas } & 1 \\ & \text { Exigencias cognitivas } & 1 \\ \text { Exigencias Psicológicas (EPS) } & \text { Exigencias sensoriales } & 1 \\ & \text { Exigencias emocionales } & 1 \\ & \text { Exigencias de esconder emociones } & 1 \\ & \text { Subtotal } & 5 \\ & \text { Influencia } & 1 \\ & \text { Control sobre el tiempo de trabajo } & 1 \\ \text { Trabajo activo y posibilidades } & \text { Posibilidades de desarrollo en el trabajo } & 1 \\ \text { de desarrollo (TA) } & \text { Sentido en el trabajo } & 1 \\ & \text { Integración en la empresa } & 1 \\ & \text { Subtotal } & 5 \\ \text { Apoyo social de la empresa y } & \text { Claridad de rol } & 1 \\ \text { calidad de liderazgo (ASL) } & \text { Calidad de liderazgo de relación con los superiores } & 1 \\ & \text { Calidad de relación con los compañeros } & 1 \\ & \text { de trabajo } & 1 \\ & \text { Subtotal } & 1 \\ \text { Compensaciones (COM) } & \text { Estima } & \text { Inseguridad respecto al contrato de trabajo } \\ & \text { Inseguridad respecto a las caracteristicas del trabajo } & 1 \\ \text { Doble presencia (DP) } & \text { Subtotal } & 3 \\ \text { Total } & \text { Preocupación por las tareas domésticas } & 2 \\ & & 20\end{array}$

\section{Análisis estadístico}

Para contrastar el modelo factorial propuesto en el SUSESO/ ISTAS-21 versión breve se realizó un análisis factorial confirmatorio (AFC), con cinco dimensiones o factores latentes que representas los factores de riesgos psicosociales expuestos en la Tabla 2, los que están asociados con sus respectivos items y sus errores de medida.

El análisis inicial examinó la normalidad multivariante de los indicadores. Los resultados del coeficiente de curtosis multivariado (coeficiente de Mardia = 104,50) indicó que la muestra presentaba una distribución no-normal. ${ }^{24}$

Ante los cuidados que se deben mantener al usar escalas tipo Likert en la elección del método para la obtención de parámetros de un AFC asociados a la normalidad ${ }^{25}$ y el nivel de medida ordinal ${ }^{25,26}$, se opta por el método Unweighted Least Squares (ULS) o Mínimos Cuadrados no Ponderados, método de estimación que no requiere de una distribución determinada; recomendado para variables categóricas y ordinales ${ }^{27,28}$, además presenta algunas ventajas en la extracción de factores más débiles. Para ejecutar el procedimiento estadístico se empleó el programa AMOS versión ${ }^{22.29}$ 
Como medidas de bondad del ajuste del modelo se emplearon criterios múltiples ${ }^{30}$ tales como: el índice de error cuadrático medio RMR (root mean square residual) ${ }^{31}$, el índice de bondad de ajuste GFI (goodness-of-fit index) ${ }^{3.31}$, el índice ajustado de bondad ajuste AGFI (adjusted goodness-of-fit index) ${ }^{31-33}$, el índice normado de ajuste NFI (normed fit index); el índice de bondad de ajuste de parsimonia PGFI (parsimony goodness-of-fit index). ${ }^{31}$ La validez convergente de los reactivos se evaluó en función de la significación estadística de las cargas factoriales de los indicadores de cada factor de riego psicosocial.

Para el la obtención de la fiabilidad del instrumento se emplea el Coeficiente 0 mega ${ }^{34}$ dado que no depende del número de ítems, ni del número de categorías de respuesta ${ }^{34}$, además es apropiado para mediciones que transgreden el supuesto de tauequivalencia; ${ }^{34,35}$ dicho coeficiente se obtuvo empleando paquete MBESS ${ }^{36}$ en R. ${ }^{37}$ La validez discriminante se evaluó empleando una matriz que compara la raíz cuadrada de la varianza media extraída (AVE) con la correlación entre constructos. ${ }^{38}$

\section{RESULTADOS}

\section{Análisis factoriales confirmatorios}

Los índices de ajuste del análisis factorial confirmatorio mostraron que el modelo se ajusta $\mathrm{GFI}=0,967, \mathrm{AGFI}=0,957, \mathrm{NFI}=0,30$; $\sin$ embargo, hay criterios que están fuera de parámetro $R M R=0,068$, PGFI $=0,737$, dando cuenta de una diferencia entre las varianzas y covarianzas del modelos. ${ }^{39}$

La Tabla 3 muestra los residuos normalizados del modelo, se presentan 39 potencialmente significados (superan |2,58|) 40 lo que excede el 5\% admitido.

Validez convergente y fiabilidad de las medidas

En la Tabla 4 se observan cargas factoriales estandarizadas $(\lambda 1)$, no se observan cargas menores a 0,3; no obstante, están en el umbral mínimo de significación $(>0,3) .^{40}$
Los cálculos de confiabilidad postulan que el índice $\omega$ debe de estar entre 0,7 y 0,934,35, por lo que sólo Apoyo social de la empresa y Calidad de liderazgo (ASL) cumple con los mínimos establecidos; si se ocupan criterios más laxos, los cuales postulan que el mínimo admisible es 0,$65 ;^{34-41}$ a la dimensión anterior se le suma Exigencias Psicológicas.

\section{Tabla 4.}

Cargas estandarizadas $(\lambda 1)$ y no estandarizadas $(\lambda 2)$, y Coeficiente omega $(\omega)$ con intervalo de confianza.

\begin{tabular}{|c|c|c|c|c|c|}
\hline Dimensión & Ítem & $\lambda 1$ & $\lambda 2$ & $\omega$ & $95 \%$ IC \\
\hline \multirow[t]{6}{*}{ Exigencias Psicológicas (EPS) } & & & & 0,675 & {$[0,645,0,702]$} \\
\hline & EPS1 & 0,508 & 1 & & \\
\hline & EPS2 & 0,32 & 0,778 & & \\
\hline & EPS3 & 0,763 & 0,953 & & \\
\hline & EPS4 & 0,733 & 2,027 & & \\
\hline & EPS5 & 0,306 & 0,852 & & \\
\hline \multicolumn{4}{|c|}{ Trabajo activo y posibilidades de desarrollo (TA) } & 0,527 & {$[0,472,0,573]$} \\
\hline & TA1 & 0,36 & 1 & & \\
\hline & TA2 & 0,327 & 0,699 & & \\
\hline & TA3 & 0,622 & 0,469 & & \\
\hline & TA4 & 0,47 & 0,709 & & \\
\hline & TA5 & 0,482 & 0,943 & & \\
\hline \multicolumn{4}{|c|}{ Apoyo social de la empresa y calidad de liderazgo (ASL) } & 0,758 & {$[0,734,0,781]$} \\
\hline & ASL1 & 0,502 & 1 & & \\
\hline & ASL2 & 0,53 & 0,359 & & \\
\hline & ASL3 & 0,706 & 2,024 & & \\
\hline & ASL4 & 0,544 & 0,383 & & \\
\hline & ASL5 & 0,75 & 2,115 & & \\
\hline \multirow[t]{4}{*}{ Compensaciones (COM) } & & & & 0,598 & {$[0,553,0,635]$} \\
\hline & COM1 & 0,368 & 1 & & \\
\hline & COM2 & 0,636 & 0,512 & & \\
\hline & COM3 & 0,658 & 0,607 & & \\
\hline \multirow[t]{3}{*}{ Doble presencia (DP) } & & & & 0,624 & {$[0,572,0,668]$} \\
\hline & DP1 & 0,784 & 1 & & \\
\hline & DP2 & 0,579 & 0,674 & & \\
\hline
\end{tabular}

Tabla 3.

Residuos normalizados del modelo.

\begin{tabular}{|c|c|c|c|c|c|c|c|c|c|c|c|c|c|c|c|c|c|c|c|}
\hline & DP2 & DP1 & СOM3 & COM2 & COM1 & ASL5 & ASL4 & ASL3 & ASL2 & ASL1 & TA5 & TA4 & TA3 & TA2 & TA1 & EPS5 & EPS4 & EPS3 & EPS2 EPS1 \\
\hline DP2 & 0 & & & & & & & & & & & & & & & & & & \\
\hline DP1 & 0 & 0 & & & & & & & & & & & & & & & & & \\
\hline сом3 & $-0,363$ & $-3,558$ & 0 & & & & & & & & & & & & & & & & \\
\hline соM2 & 2,059 & 0,509 & $-3,248$ & 0 & & & & & & & & & & & & & & & \\
\hline COM1 & 2,524 & 0,201 & $-0,162$ & 6,222 & 0 & & & & & & & & & & & & & & \\
\hline ASL5 & $-0,069$ & $-0,999$ & 4,095 & $-0,411$ & $-3,577$ & 0 & & & & & & & & & & & & & \\
\hline ASL4 & $-0,392$ & 0,355 & 0,793 & $-0,51$ & $-2,622$ & $-0,634$ & 0 & & & & & & & & & & & & \\
\hline ASL3 & $-0,713$ & $-0,877$ & 4,018 & $-2,36$ & $-2,875$ & 4,713 & 0,008 & 0 & & & & & & & & & & & \\
\hline ASL2 & 2,571 & 0,42 & 0,08 & 0,124 & $-0,115$ & $-2,452$ & $-0,403$ & $-3,47$ & 0 & & & & & & & & & & \\
\hline ASL5 & 0,553 & 0,231 & $-0,224$ & 0,659 & $-0,204$ & $-0,005$ & $-0,285$ & $-0,56$ & $-0,455$ & 0 & & & & & & & & & \\
\hline TA5 & $-0,247$ & $-0,605$ & 2,381 & $-0,273$ & $-0,264$ & $-0,052$ & $-0,263$ & 0,32 & 0,937 & 5,409 & 0 & & & & & & & & \\
\hline TA4 & $-0,372$ & 0,537 & $-0,154$ & $-0,375$ & $-2,306$ & $-0,271$ & $-0,22$ & $-0,044$ & 0,065 & 5,105 & 6,324 & 0 & & & & & & & \\
\hline TA3 & 0,763 & 0,366 & 0,742 & $-0,572$ & $-2,744$ & $-0,373$ & $-0,384$ & 0,749 & $-3,01$ & $-0,053$ & 0,924 & 3,431 & 0 & & & & & & \\
\hline TA2 & $-2,047$ & $-0,159$ & 0,868 & $-0,154$ & 0,806 & $-0,374$ & 0,285 & 0,249 & $-0,563$ & $-0,594$ & $-2,916$ & $-5,339$ & $-0,701$ & 0 & & & & & \\
\hline TA1 & $-0,04$ & 0,042 & 0,858 & $-0,384$ & 0,393 & $-0,558$ & 0,915 & 0,898 & $-2,871$ & 2,104 & $-2,774$ & $-0,036$ & 0,089 & 0,714 & 0 & & & & \\
\hline EPS5 & 0,668 & 0,395 & $-0,142$ & 0,668 & $-0,12$ & $-0,912$ & $-0,323$ & $-0,151$ & 0,416 & $-2,838$ & $-4,633$ & $-5,591$ & $-3,282$ & 4,905 & 0,676 & 0 & & & \\
\hline EPS4 & $-0,745$ & $-0,972$ & $-0,687$ & 0,004 & 0,27 & 0,264 & 0,81 & $-0,178$ & 3,592 & $-0,231$ & $-0,407$ & $-0,569$ & 0,271 & 2,662 & 0,182 & 0,767 & 0 & & \\
\hline EPS3 & $-0,86$ & 0,489 & $-0,927$ & 0,075 & $-0,465$ & $-0,803$ & 0,103 & $-0,914$ & 3,219 & $-0,47$ & 0,515 & $-0,697$ & 0,032 & $-0,298$ & 0,578 & 0,017 & $-0,86$ & 0 & \\
\hline EPS2 & $-0,643$ & 0,676 & $-0,34$ & 0,186 & $-0,434$ & $-2,508$ & $-0,513$ & $-0,163$ & 4,675 & $-0,688$ & $-2,415$ & $-4,581$ & $-3,66$ & $-0,097$ & $-4,446$ & 3,963 & 0,53 & 4,341 & 0 \\
\hline EPS1 & $-0,238$ & $-0,661$ & 0,588 & 0,182 & $-0,562$ & 0,615 & 0,151 & $-0,868$ & 2,887 & 3,942 & 2,444 & 2,767 & 0,587 & 0,607 & 3,126 & $-2,48$ & $-0,796$ & $-0,498$ & $\begin{array}{ll}-3,638 & 0\end{array}$ \\
\hline
\end{tabular}

Nota: Los Residuos > 2.58| están en negritas. DP= Doble Presencia; Com= Compensaciones; APS= Apoyo social de la empresa y calidad de liderazgo;

$\mathrm{TA}=$ Trabajo activo y posibilidades de desarrollo; EPS= Exigencias Psicológicas. 
Tabla 5.

Correlaciones cuadráticas entre factores y varianza extraída media (en diagonal).

$\begin{array}{lccccc}\text { Dimensiones } & \text { EPS } & \text { TA } & \text { ASL } & \text { COM } & \text { DP } \\ \text { Exigencias psicológicas (EPS) } & 0,315 & 0,212 & 0,462 & 0,353 & 0,453 \\ \begin{array}{l}\text { Trabajo activo y posibilidades de } \\ \text { desarrollo (TA) }\end{array} & 0,215 & 0,593 & 0,507 & 0,128 \\ \begin{array}{l}\text { Apoyo social de la empresa y calidad } \\ \text { de liderazgo (ASL) }\end{array} & & 0,378 & 0,587 & 0,100 \\ \text { Compensaciones (COM) } & & & 0,324 & 0,245 \\ \text { Doble presencia (DP) } & & & & 0,475\end{array}$

\section{Validez Discriminante}

Para comprobar este tipo de validez, se emplea la matriz de la varianza media extractada con la correlación al cuadrado entre todos los constructos ${ }^{38}$, que se puede ver en la Tabla 5. Para que exista validez discriminante entre constructos, es necesario que la varianza media extractada sea superior a la correlación al cuadrado entre los componentes del constructo.

$\mathrm{Al}$ revisar los resultados de la Tabla 5, se puede afirmar que la validez discriminante entre la dimensión Trabajo activo y posibilidades de desarrollo y Apoyo social de la empresa y calidad de liderazgo es cuestionable; además, las varianzas medias extractadas están por debajo de 0,5 , que es el parámetro espetado para cada dimensión. ${ }^{40}$

\section{DISCUSIÓN}

Las dificultades de medición del cuestionario SUSESO/ISTAS-21 entorpecen la toma de decisiones acertadas y eficaces por parte de las organizaciones en Chile, tendiendo al sesgo e inflación de los resultados obtenidos. Esto es resultado de los problemas de validación en las propiedades psicométricas del instrumento. ${ }^{42,43}$
La creación de una versión reducida del SUSESO/ISTAS-21 responde a una necesidad de implementación en los sistemas de gestión de cada empresa ${ }^{13}$, dado que reduce los tiempos de aplicación del instrumento, minimizando el impacto en la productividad de la empresa. ${ }^{44}$ No obstante, en el caso del proceso de adaptación o validación, no se pueden transferir las propiedades de validez y confiabilidad de la versión extensa a la breve; ${ }^{12}$ esta versión del Cuestionario SUSESO/ISTAS-21 fue construida empleando como criterio de selección las cargas factoriales más altas $^{45}$, procedimiento que no está exento de sesgos de selección. ${ }^{44}$ El actual empleo del SUSESO/ISTAS-21 en su versión breve, en función de los análisis anteriores, y en ausencia de las propiedades psicométricas para el conocimiento público es cuestionable, dado que no cuenta con la evidencia empírica que respalde el modelo teórico propuesto; además, la estrategia de reducción de dimensiones, la cual pretende obtener valores de escala a partir de la sumatoria de los ítems relacionadas con cada factor de riesgo, no es apropiada.

Sólo dos dimensiones están por encima de los estándares de confiabilidad esperados, evidenciando los problemas al momento de las mediciones en las empresas. Cabe señalar que, ante estos descubrimientos, las pequeñas y medianas empresas (PyME) tienden a una inversión del capital humano erróneo y un gasto inadecuado de los recursos económicos ${ }^{46}$, limitando el desarrollo productivo en esfuerzos poco favorables ${ }^{47}$, así como un menoscabo de la importancia en la prevención de salud en la prevención de riesgos. ${ }^{48}$

Por tanto, se pone en manifiesto la necesidad en la mejora de las propiedades psicométricas actuales del cuestionario para la vigilancia de RPS, fortaleciendo la creciente cultura preventiva en materia de salud mental en el trabajo y contextos organizacionales. 
1. Oficina Internacional del Trabajo (OIT). Cambios en el mundo del trabajo; Memoria del Director General. Ginebra: OIT; 2006.

2. Messenger J, Ghosheh N. Deslocalización y condiciones laborales del trabajo a distancia [en línea]. España: Ministerio de Trabajo e Inmigración; 2011. [consultado 22 oct 2017]. Disponible en: http://www.ilo.org/global/publications/ilobookstore/order-online/books/WCMS_166084-/lang--es/index.htm

3. Aranda C, Pando M, Ronquillo JL. Confiabilidad y validez de una guía de identificación sobre factores psicosociales en trabajadores mexicanos de diversos giros laborales. Rev iberoamer psicol: cien tec. 2014;7(1):25-38.

4. Oficina Internacional del Trabajo (OIT). Informe Mundial sobre Salarios 2016/2017 Desigualdad salarial en el lugar de trabajo. Ginebra: OIT; 2016.

5. Organización Internacional del Trabajo (OIT). Lista de enfermedades profesionales de la OIT; revisada en 2010. Ginebra: OIT SafeWork; 2010.

6. Organización Internacional del Trabajo (OIT). Estrés en el trabajo un reto colectivo. Día mundial de la seguridad y la salud en el trabajo. Ginebra: OIT; 2016.

7. Vives A, Molina A, Gray N, González F. Envejecimiento y trabajo en Chile: propuesta para el monitoreo de la salud laboral. En: Irarrázaval I, Piña E, Letelier M. Propuestas para Chile; Concurso de políticas públicas 2016. Santiago: Pontificia Universidad Católica de Chile; 2017. p. 17-49.

8. Ministerio del Trabajo. Normas sobre accidentes del Trabajo y Enfermedades Profesionales. Santiago de Chile: Ministerio del Trabajo; 1968.

9. Dirección Nacional del Servicio Civil. Propuesta de estrategia para la medición e intervención de las condiciones laborales en los servicios públicos de la administración central del estado. Santiago de Chile: Dirección Nacional del Servicio Civil; 2012.

10. Larrañaga 0. Inequality, Poverty and Social Policy: Recent Trends in Chile. Paris: OECD; 2009. 35 p. (OECD Social, Employment and Migration Working Papers; 85)

11. Alvarado $R$, Nella $M$, Villalón $M$, Hirmas $M$, Pastorino $M S$. Adaptación y análisis psicométrico de un cuestionario para evaluar riesgos psicosociales en el trabajo en Chile: versión media del CoPsoO. Rev Chil Salud Pública. 2009; 13(1): 7-16.

12. Alvarado R, Pérez-Franco J, Saavedra N, Fuentealba C, Alarcón A, Marchetti N, Aranda W. Validación de un cuestionario para evaluar riesgos psicosociales en el ambiente laboral en Chile. Rev Med Chile. 2012; 140(9):1154-1163.

13 Kompier M. Assessing the psychosocial work environment - "subjective" versus "objective" measurement. Scand. J Work Environ Health. 2005; 31(6):405-408.

14. Kristensen T, Borg V. Copenhagen Psychosocial Questionnaire (COPSOQ). Copenhagen: Det Nationale Forskningscenter for Arbejdsmiljø; 2003.

15. Kristensen T, Hannerz H, Høgh A, Borg V. The Copenhagen Psychosocial Questionnaire; a tool for the assessment and improvement of the psychosocial work environment. Scand. J Work Environ Health. 2005; 31(6):438-449.

16. Moncada S, Llorens $C$, Kristensen T. Manual para la evaluación de riesgos psicosociales en el trabajo. Barcelona: Instituto Sindical de Trabajo, Ambiente y Salud; 2002.

17. Acevedo G, Sánchez J, Farias M, Fernández A. Riesgos Psicosociales en el Equipo de Salud de Hospitales Públicos de la Provincia de Córdoba, Argentina. Cienc Trab. 2013; 15(48):140-147.

18. Ministerio de Trabajo, Empleo y Seguridad Social-Superintendencia de Riesgos del Trabajo. Estudio de adaptación y validación para Argentina del Cuestionario Psicosocial de Copenhague (CoPsoQ-ISTAS21). Buenos Aires: Universidad Nacional de Avellaneda-Ministerio de Trabajo, Empleo y Seguridad Social; 2015.

19. Charria V, Sarsosa K, Arenas F. Factores de riesgo psicosocial laboral: métodos e instrumentos de evaluación. Rev Fac Nac Salud Pública. 2011; 29(4): 380-391.

20. Fernández-Arata JM, Calderón-De la Cruz G, Navarro-Loli JS. Psicología de la Salud Ocupacional: Una especialidad emergente en el Perú. Rev Med Hered. 2016; 27(3):193-194.

21. Pando $M$, Varillas $W$, Aranda $C$, Elizalde $F$. Análisis factorial exploratorio del Cuestionario de factores psicosociales en el trabajo en Perú. An Fac Med (Lima). 2016; 77(4):365-371.

22. Bulger CA, Matthews RA, Hoffman ME. Work and personal life boundary management: Boundary strength, work/personal life balance, and the segmentationintegration continuum. J Occup Health Psychol. 2007; 12(4):365-375.
23. Aranda B, Barraza J, Romero J, Quiñonez L, Ceniceros A, González G, Esparza J. Factores psicosociales y sindrome de Burnout en médicos generales de Tepic, Nayarit (México). Salud (Barranquilla). 2015; 31(2):245-254.

24. Mardia KV. Applications of some measures of multivariate skewness and kurtosis in testing normality and robustness studies. Sankhya. 1974; 36(2):115-128.

25. Flora DB, Curran PJ. An Empirical Evaluation of Alternative Methods of Estimation for Confirmatory Factor Analysis with Ordinal Data. Psychol Methods. 2004; 9(4):466-491.

26. Coenders G, Saris E. Categorization and Measurement Quality. The Choice between Pearson and Polychoric Correlations. En: Saris WE, editor. The multitratit-multimethod approach to evaluate measurement instruments. Budapest: Eötvös University Pres; 1995. p. 125-144.

27. Brown TA. Confirmatory factor analysis for applied research. New York: Guilford Press; 2006.

28. Schumacker RE, Lomax RG. A beginner's guide to structural equation modeling. 2a ed. New Jersey: Lawrence Erlbaum Associates Publishers; 2004.

29. Arbuckle JL. Amos (Version 22.0) [software]. Chicago: IBM SPSS; 2013.

30. Escobedo MT, Hernández JA, Estebané V, Martínez G. Modelos de ecuaciones estructurales: Características, fases, construcción, aplicación y resultados. Cienc trab. 2016; 18(55):16-22.

31. Arbuckle JL. Amos 18 user's guide. Chicago: Amos Development Corporation; 2009.

32. Martínez MC. Orientación a mercado y aprendizaje organizacional: evidencia empírica en el sector empresarial de Aguascalientes. Invest Cienc: UAA. 2002; 27:33-38.

33. Mulaik SA, James LR, Van Alstine J, Bennett N, Lind S, Stilwell, CD. Evaluation of Goodness-of-Fit Indices for Structural Equation Models. Psychol Bull. 1989; 105(3):430-445.

34. Ventura-León J, Caycho-Rodríguez T. El coeficiente Omega: un método alternativo para la estimación de la confiabilidad. Rev latinoam cienc soc niñez juv. 2007; 15(1):625-627.

35. Dunn T, Baguley T, Brunsden V. From alpha to omega: A practical solution to the pervasive problem of internal consistency estimation. Br J Psychol. 2014; 105(3):399-412.

36. Kelley K. The MBESS R Package. Notre Dame, IN: University of Notre Dame; 2018.

37. $\mathrm{R}$ Development Core Team. R: A language and environment for statistical computing [online]. Vienna: R Foundation; 2012 [cited 2018 Abr 13]. Available from: http://www.R-project.org/

38. Fornell C, Larcker D. Evaluating Structural Equation Models with Unobservable Variables and Measurement Error. J Mark Res. 1981; 18(1):39-50.

39. Arbuckle J. Amos 6.0 user's guide. Chicago: Amos Development Co.; 2003.

40. Hair J, Anderson R, Tatham R, Black W. Análisis Multivariante. 5a ed. Madrid: Prentice Hall; 1999.

41. Katz M. Multivariable Analysis: A Practical Guide for Clinicians and Public Health Researchers. Cambridge: Cambridge University Press; 2006.

42. Furr RM, Bacharach VR. Psychometrics: An introduction. Thousand Oaks, CA: Sage; 2013.

43. Sigerson L, Cheng C. Scales for measuring user engagement with social network sites: A systematic review of psychometric properties. Comput Hum Behav. 2008; 83(1):87-105.

44. Varela J, Prat R, Voces $C$, Rial A. Una nueva escala para la evaluación de la calidad de los servicios de hosteleria. Psicothema. 2006; 18(1):135-142

45. Candia M, Pérez-Franco J, González D. Manual del Método del Cuestionario SUSESO/ISTAS 21 versiones completa y breve. Santiago de Chile: Superintendencia de Seguridad Social; 2016.

46. Nourani M, Chandra V, Long Q, Lu W. Measuring Human, Physical and Structural Capital Efficiency Performance of Insurance Companies. Soc Indic Res. 2017; 137(1):281-315

47. Villalobos G, Pedroza R. Perspectiva de la teoria del capital humano acerca de la relación entre educación y desarrollo económico. Tiempo Educar. 2009; 10(20):273-306.

48. Rial E. Las prioridades de la investigación sobre seguridad y salud en el trabajo: el desafio para una Europa de 27. Arch Prev Riesgos Labor. 2006; 9(2): 56-59. 